\title{
Effect of Rosiglitazone on Liver Structure and Function in Genetically Diabetic Akita Mice
}

\author{
Bianca Hemmeryckx ${ }^{1}$, Marijke Gaekens ${ }^{1}$, David J. Gallacher ${ }^{2}$, Hua Rong Lu ${ }^{2}$ and Henri Roger Lijnen ${ }^{1}$ \\ ${ }^{1}$ Center for Molecular and Vascular Biology, Department of Cardiovascular Sciences, KU Leuven, Leuven, Belgium and ${ }^{2}$ Translational Sciences, \\ Safety Pharmacology Research, Janssen Research \& Development, Janssen Pharmaceutical NV, Beerse, Belgium
}

(Received 8 March 2013; Accepted 17 June 2013)

\begin{abstract}
Genetically diabetic Akita mice, kept on a high-fat and high-cholesterol diet, and treated with the peroxisome proliferator-activated receptor- $\gamma$ agonist rosiglitazone $(10 \mathrm{mg} / \mathrm{kg}$ per day during 4 months), displayed rosiglitazone-induced side effects, similar to those observed in patients, including weight and fat gain and early signs of hypertrophic cardiomyopathy. As several cases of hepatotoxicity were reported in patients receiving rosiglitazone treatment, this study evaluated whether rosiglitazone also induced hepatotoxicity in these diabetic animals. Liver structure and function was analysed in wild-type and rosiglitazone-treated and untreated Akita mice, kept for 4 months on the high-fat and high-cholesterol diet. Decreased circulating levels of the liver enzymes aspartate and alanine aminotransferase and increased levels of alkaline phosphatases were observed upon rosiglitazone treatment, whereas liver weight was markedly increased. Rosiglitazone administration was associated with liver steatosis, as demonstrated by triglyceride accumulation. However, gene expression of steatosis markers in liver tissue was not markedly affected by rosiglitazone treatment, while expression of fatty acid transport protein was reduced by rosiglitazone treatment, suggesting an impairment of the fatty acid $\beta$-oxidation pathway. mRNA expression of pro- and anti-oxidant enzymes and liver 3-nitrotyrosine content was not affected. Furthermore, gene and protein expression of macrophage markers and of cell adhesion molecules did not indicate progression to steatohepatitis, whereas an unaltered collagen deposition did not suggest steatofibrosis. In conclusion, rosiglitazone treatment of diabetic Akita mice induced liver steatosis without, however, progression to more advanced stages of liver disease.
\end{abstract}

Insulin resistance is an important cause of non-alcoholic fatty liver disease, one of the most common forms of chronic liver disease [1-5]. Thiazolidinediones, including rosiglitazone, are peroxisome proliferator-activated receptor- $\gamma$ (PPAR- $\gamma)$ agonists that improve diabetes by increasing tissue sensitivity for insulin [6]. However, large-scale clinical use has revealed serious side effects such as water retention, cardiovascular toxicity and weight gain [7]. The molecular mechanisms underlying these complications are poorly understood, to some extent due to the lack of suitable diabetic animal models. Pre-clinical models in rodents indeed appeared to have only limited predictive power in respect to the observed clinical adverse effects associated with rosiglitazone [8]. Next to drug-induced cardiac toxicity, drug-induced liver injury is the second largest cause for the withdrawal of drugs from the market or for stopping the drug development process in its early-phase, thus causing huge financial losses [9]. The mechanisms of druginduced liver injury are not fully clear, especially the mechanism by which rosiglitazone caused liver toxicity [10,11]. Common manifestations of drug-induced liver injury include lipid storage disorders, such as steatosis or phospholipidosis. Drug-induced steatosis, characterized by an increased accumulation of lipids in the form of triglycerides, is a serious issue for pharmaceutical companies as it could lead to liver failure,

Author for correspondence: H. Roger Lijnen, Center for Molecular and Vascular Biology, KU Leuven, Campus Gasthuisberg, CDG, Herestraat 49, Box 911, B-3000 Leuven, Belgium (fax 32-16-345990, e-mail: roger.lijnen@med.kuleuven.be). resulting in the withdrawal of the drug from the market $[12,13]$. In this study, we have used the Ins $2^{\text {Akita }}$ mouse to evaluate the hepatotoxic effect of rosiglitazone. The Akita mice have a spontaneous point mutation ( $\mathrm{Cys}^{96}$ to $\mathrm{Tyr}$ ) in the insulin 2 gene, leading to accumulation of misfolded pro-insulin in the endoplasmatic reticulum and eventually to apoptosis of $\beta$-cells and failure to secrete mature insulin [14,15]. These mice develop hyperglycaemia, hypoinsulinaemia, polydipsia and polyuria and are frequently used to study complications associated with type 1 diabetes. They also develop type 2 diabetes with insulin resistance in skeletal muscle, liver and brown adipose tissue that is partly due to hyperglycaemia $[14,15]$. Therefore, the Akita mouse is frequently used as a model for both type 1 and type 2 diabetes. In a previous study, rosiglitazone administration to Akita mice fed a high-fat and high-cholesterol diet for 4 months, showed body-weight and fat mass gain and early signs of hypertrophic cardiomyopathy [16]. As liver disease is associated with insulin resistance, and hepatotoxicity and liver failure are potential side effects of rosiglitazone treatment, we have studied the potential mechanisms of rosiglitazone in different stages of non-alcoholic fatty liver disease in the genetically diabetic Akita mouse.

\section{Materials and Methods}

Animal model. Male Akita mice (C57B1/6J genetic background) were purchased from The Jackson Laboratories (Bar Harbor, MN, USA). Mice were kept in individual micro-isolation cages on a 12-hr day/ night cycle at $20-22^{\circ} \mathrm{C}$ and exposed ad libitum to water and regular 
chow. From 10-week-old mice, plasma was prepared after an overnight fasting by collecting blood from the retro-orbital sinus on $3.8 \%$ trisodium citrate. The animals were then put on a high-fat and high-cholesterol diet (19.5\% fat and 1.25\% cholesterol; E15751-30, Sniff Spezialitäten GmbH, Soest, Germany) for 4 months. Male wildtype C57B1/6J mice $(n=6)$ were also kept on the high-fat and highcholesterol diet for comparison. Akita mice were divided into two groups $(\mathrm{n}=6$ each): one group received the high-fat and highcholesterol diet with rosiglitazone $(10 \mathrm{mg} / \mathrm{kg}$ body-weight per day), while the control group received only the high-fat and high-cholesterol diet. Rosiglitazone was custom made by Apin Chemicals Limited (Oxon, UK). This dosage regimen of rosiglitazone was based on previous experiments in rodents [16,17]. After 4 months, plasma was collected and the mice were killed with an i.p. injection of $60 \mathrm{mg} / \mathrm{kg}$ sodium pentobarbital (Nembutal, Abbott Laboratories, North Chicago, IL, USA). After a saline perfusion step to remove blood from all organs, the washed liver was removed, weighed and divided in portions for histological analysis, RNA extraction and biochemical analyses. All the animal procedures were approved by the Ethical Committee of the KU Leuven and performed in accordance with the NIH Guide for the Care and Use of Laboratory Animals (1996).

Histology and immunohistochemistry. Liver tissue was fixed with $1 \%$ paraformaldehyde in phosphate-buffered saline for $20 \mathrm{hr}$ at $4^{\circ} \mathrm{C}$, paraffin-embedded and prepared as cross sections of $8 \mu \mathrm{m}$. Liver sections were stained with haematoxylin and eosin (H\&E) and with Sirius Red (SR) as described previously [18]. Liver sections were also deparaffinized, rehydrated and used for immunohistochemical detection of 3-nitrotyrosine (3-NT), F4/80, Mac-3, intercellular adhesion molecule-1 (ICAM-1), vascular cell adhesion molecule-1 (VCAM-1) and cytokeratin-19 (CK-19) antigen. Antigens for the 3NT, F4/80 and Mac-3 stainings were exposed by incubating sections with an antigen retrieval solution (DAKO, Heverlee, Belgium) according to the manufacturer's instructions. To expose CK-19 antigens, liver sections were incubated for $7 \mathrm{~min}$. with trypsin $(25 \mu \mathrm{g} /$ $\mathrm{ml}$ in $0.1 \% \mathrm{CaCl}_{2}$ ). All sections were then incubated for $20 \mathrm{~min}$. with methanol supplemented with $0.3 \% \mathrm{H}_{2} \mathrm{O}_{2}$ at room temperature to block endogenous peroxidase activity. A specific binding to secondary antibodies (Abs) was prevented by incubating sections with $20 \%$ preimmune rabbit serum (45 min., room temperature, X0902, DAKO) for the F4/80, Mac-3, ICAM-1 and VCAM-1 stainings or with $20 \%$ or 5\% pre-immune goat serum (45 min., room temperature, X0907, DAKO) for the 3-NT and CK-19 staining, respectively. Primary and secondary Abs were diluted in Tris-NaCl-blocking buffer and incubated at room temperature: primary $\mathrm{Abs}$ were rabbit antinitrotyrosine $(1 / 1000,1 \mathrm{hr}$, 06-284, Millipore, 25 Billerica, MA, USA), rat anti-mouse F4/80 (1/100, overnight (ON), MCAP497, AbD Serotec, Düsseldorf, Germany), rat anti-mouse Mac-3 (1/50, ON, 553322, Pharmingen, San Diego, CA, USA), goat anti-mouse ICAM-1 (1/250, ON, AF796, R\&D Systems, Minneapolis, MN, USA), goat anti-mouse VCAM-1 (1/100, ON, AF643, R\&D Systems) or rabbit anti-mouse CK-19 (1/100, ON, ab15463, Abcam, Cambridge, UK) followed by secondary Abs horseradish peroxidase (HRP)-labelled goat anti-rabbit (GAR) Ab (1/100, 45 min., P0448, DAKO) for the 3NT staining, biotinylated (B) rabbit anti-rat antibody (1/300, $45 \mathrm{~min}$., E0468, DAKO) pre-incubated with $10 \%$ pre-immune mouse serum (M5905, Sigma-Aldrich, St. Louis, MO, USA) for F4/80 and Mac-3 antigen visualization, rabbit anti-goat-B antibody (1/300, $45 \mathrm{~min}$., E0466, DAKO) preincubated with $10 \%$ pre-immune mouse serum for the ICAM-1 and VCAM-1 stainings, and GAR-B antibody (1/300, 45 min., E0432, DAKO) for the CK-19 staining. For signal amplification, the Tyramide Signal Amplification Cyanine 3 System (Perkin Elmer, Boston, MA, USA) (F4/80, Mac-3, CK-19) or streptavidin-HRP (Perkin Elmer) in Tris-NaCl-blocking buffer (1/100, 30 min., room temperature) (ICAM-1, VCAM-1) was used. For the 3-NT, ICAM-1, VCAM-1 and CK-19 stainings 3, 3-diaminobenzidine were applied for detection with Harris counterstaining (ICAM-1, VCAM-1). Sections were dehydrated and mounted with dibutyl phthalate xylene (3-NT, ICAM-1, VCAM-1, CK-19) or mounted with Prolong Gold with 4',6-diamidino-2-phenylindole (DAPI, Life Technologies, Carlsbad, CA, USA) (F4/80) or Vectorshield with DAPI (Vector Laboratories, Inc., Burlingame, CA, USA) (Mac-3).

Microscopy analysis. H\&E, SR, Mac-3, 3-NT, ICAM-1 and VCAM-1 stainings were analysed with a Zeiss AxioVert 200M microscope (Zeiss, Jena, Germany), while images of F4/80-stained liver sections were taken using an AxioPlan 2 Imaging microscope (Zeiss). Pictures were taken at random (minimal 4 images per liver section, 2-4 sections per liver) and analysed through computer-assisted image analysis (AxioVision Rel 4.8, Zeiss) at $400 \times$ magnification. The amount of macrophages (F4/80, Mac-3), adhesion molecules (ICAM1, VCAM-1), fibrosis (SR) or oxidative stress (3-NT) in the liver was determined by measuring both stained (Cy3 for Mac-3 and F4/80; 3 diaminobenzidine for other stainings) and total area of the analysed image and was subsequently expressed as percentages of stained area to total analysed area. For each staining, all measurements for each animal were averaged, and subsequently, data for all animals in one group were averaged. The presence of bile duct hyperplasia after drug-induced cholestasis in the periportal region of the acinus in each liver section was investigated by immunohistochemical detection of the bile duct epithelial cell antigen CK-19. Two liver sections per animal were examined for an increased number of CK-19 positive biliary ductules in each acinus. When the number of CK-19 positive biliary ductules in each acinus exceeded the number 10, biliary duct hyperplasia was present. Per section, the frequency of the appearance of biliary duct hyperplasia was counted and averaged per animal. Subsequently, data for all animals in one group were averaged.

Gene expression analysis. To monitor gene expression, quantitative real-time PCR was used. Liver tissue was homogenized with lysing matrix tubes containing ceramic and silica beads in a Hybaid Ribolyser (Hybaid Ltd., Thermo, Waltham, MA, USA). Total DNAfree RNA was extracted with the RNeasy kit (Qiagen, Valencia, CA, USA), and the concentration was determined spectrophotometrically (ND-2000, Nanodrop Technologies, Wilmington, DE, USA). To obtain cDNA, $50 \mathrm{ng}$ of total RNA was incubated with Taqman Reverse Transcription Kit reagents and random hexamers (Life Technologies) at $48^{\circ} \mathrm{C}$ for $60 \mathrm{~min}$. and for $5 \mathrm{~min}$. at $95^{\circ} \mathrm{C}$. Taqman gene expression assays and Taqman Universal PCR Master Mix (Life Technologies) were used to determine liver mRNA levels with the ABI-prism Fast 7500 detector (Table 1). PPAR- $\gamma_{1}$ and PPAR- $\gamma_{2}$ expression were monitored using primers and probes as described [16]. $\beta$-actin was used as housekeeping gene; its stable expression was verified throughout the experiments. Data were obtained as cycle threshold $(\mathrm{Ct})$ values and were normalized to the housekeeping gene $\left(\Delta \mathrm{Ct}=\mathrm{Ct}_{\text {target }}-\mathrm{Ct}_{\beta \text {-actin }}\right)$.

Biochemical analysis. Plasma C-reactive protein levels were measured with a commercially available ELISA (Uscn Life Science Inc., Wuhan, China). Levels of the liver enzymes alanine aminotransferase (ALT), aspartate aminotransferase (AST) and alkaline phosphatases were analysed using routine laboratory assays. The amount of triglycerides in the liver was quantified with the triglycerides FS* kit (Diasys, Holzheim, Germany), after preparation of liver tissue as described previously [19].

Statistical analysis. Data are presented as means \pm S.E.M.. Statistical differences between two groups were determined with the nonparametric Mann-Whitney $U$-test. Differences were considered significant for $p<0.05$. The statistical analyses were performed with GraphPad Prism 4.03 software (GraphPad, La Jolla, CA, USA). 
Table 1.

Genes and Taqman gene expression assays.

\begin{tabular}{|c|c|c|}
\hline Abbreviation & Name & $\begin{array}{l}\text { Taqman gene } \\
\text { expression assay }\end{array}$ \\
\hline $\begin{array}{l}\text { SREBP1c } \\
\text { or Srebf1 }\end{array}$ & $\begin{array}{l}\text { Sterol regulatory } \\
\text { element-binding protein } 1 \mathrm{c}\end{array}$ & Mm00550338_m1 \\
\hline PPAR- $\alpha$ & $\begin{array}{l}\text { Peroxisome proliferator-activated } \\
\text { receptor- } \alpha\end{array}$ & Mm00440939_m1 \\
\hline CD36 & Cluster of differentiation 36 & Mm00432403_m1 \\
\hline FAS & Fatty acid synthase & Mm00433237_m1 \\
\hline Cat & Catalase & Mm00437992_m1 \\
\hline SOD1 & Superoxide dismutase 1 & Mm01700393_m1 \\
\hline Gpx1 & Glutathione peroxidase 1 & Mm00656767_m1 \\
\hline Xdh1 & Xanthine dehydrogenase 1 & Mm00442110_m1 \\
\hline Nox-4 & NADPH oxidase-4 & Mm00479246_m1 \\
\hline iNOS & Inducible nitric oxide synthase & Mm01309902_m1 \\
\hline eNOS & Endothelial nitric oxide synthase & Mm00435217_m1 \\
\hline Arg1 & Arginase 1 & Mm00475988_m1 \\
\hline Mrc1 & Mannose receptor 1 & Mm00485148_m1 \\
\hline TNF- $\alpha$ & Tumour necrosis factor- $\alpha$ & Mm00443258_m1 \\
\hline IL-6 & Interleukin-6 & Mm00446190_m1 \\
\hline $\begin{array}{l}\mathrm{MCP}-1 \\
\text { or } \mathrm{Ccl} 2\end{array}$ & Monocyte chemotactic protein-1 & Mm00441242_m1 \\
\hline $\begin{array}{l}\mathrm{F} 4 / 80 \\
\text { or EMR1 }\end{array}$ & $\begin{array}{l}\text { EGF-like module-containing } \\
\text { mucin-like hormone } \\
\text { receptor-like } 1\end{array}$ & Mm00802529_m1 \\
\hline Csf1r & $\begin{array}{l}\text { Colony-stimulating factor } \\
1 \text { receptor }\end{array}$ & Mm01266652_m1 \\
\hline CD68 & Microsialin & Mm03047340_m1 \\
\hline ICAM-1 & Intercellular adhesion molecule-1 & Mm00516023_m1 \\
\hline VCAM-1 & $\begin{array}{l}\text { Vascular cell adhesion } \\
\text { molecule-1 }\end{array}$ & Mm01320970_m1 \\
\hline FATP & Fatty acid transport protein & Mm00449511_m1 \\
\hline MCAD & $\begin{array}{l}\text { Medium-chain acyl-CoA } \\
\text { dehydrogenase }\end{array}$ & Mm00431611_m1 \\
\hline Aktb & $\beta$-actin & Mm01205647_g1 \\
\hline
\end{tabular}

\section{Results}

Effect of genotype. After 4 months on high-fat and highcholesterol diet, body-weight of the Akita mice was significantly lower than that of the wild-type mice kept on the same diet $(19 \pm 0.94 \mathrm{~g}$ versus $31 \pm 1.1 \mathrm{~g} ; p=0.002)$. Liver weight was similar between Akita mice $(1.492 \pm 71 \mathrm{mg})$ and wild-type mice $(1.477 \pm 64 \mathrm{mg})$, corresponding to $8.0 \pm 0.21 \%$ and $4.7 \pm 0.12 \%$ of total body-weight, respectively. Food intake was comparable between both groups (not shown). Elevated levels of plasma alkaline phosphatase and C-reactive protein were observed in Akita versus wild-type mice at the start of the diet (Table 2). After 4 months of high-fat and high-cholesterol diet, untreated Akita mice showed higher plasma alkaline phosphatase, AST and ALT levels compared with wild-type controls. Less hepatic lipid accumulation was noticed in diabetic Akita versus wild-type mice by $\mathrm{H} \& \mathrm{E}$ staining (fig. 1A,B), which was confirmed by lower triglyceride levels in hepatic extracts of these mice $\left(8.2 \pm 1.7 \mathrm{mg} \times \mathrm{dL}^{-1}\right.$ / $\mathrm{mg}$ tissue versus $18 \pm 1.9 \mathrm{mg} \times \mathrm{dL}^{-1} / \mathrm{mg}$ tissue; $\left.p<0.02\right)$. Liver gene expression of the steatosis markers PPAR- $\alpha$ and $\operatorname{PPAR} \gamma 2$ was increased in diabetic Akita versus wild-type mice, while the expression of sterol regulatory element-binding protein 1c (SREBP1c), PPAR $\gamma 1$, cluster of differentiation 36 (CD36) and fatty acid synthase was not influenced by genotype

(Table 3).

Expression analysis in liver tissue of anti-oxidant genes revealed no effect of genotype of superoxide dismutase 1 (SOD1), catalase (Cat) and glutathione peroxidase 1 (Gpx1) (Table 3). Of the pro-oxidant genes analysed, only inducible nitric oxide synthase (iNOS) and endothelial NOS (eNOS) were down-regulated, whereas xanthine dehydrogenase 1 (Xdh1) and NADPH oxidase-4 (Nox-4) were not significantly affected. Staining of liver sections for 3-NT, a marker for oxidative stress, indicated no marked effect by genotype (fig. 1D,E). Quantitative analysis confirmed this finding (Table 4).

Monitoring of macrophage content in liver tissues by staining for F4/80 (fig. 1G,H) or Mac-3 (not shown) revealed a higher Mac-3 expression and a trend towards elevated F4/80 expression in diabetic Akita versus wild-type mice (Table 4). This was compatible with higher gene expression (lower $\Delta \mathrm{Ct}$ values) of the general macrophage markers F4/80, colonystimulating factor 1 receptor (Csf1r) and CD68 (Table 3). Relative gene expression of the M2 macrophage marker arginase 1 (Arg1), but not of mannose receptor 1 (Mrc1), was significantly up-regulated in Akita versus wild-type animals. In addition, the M1 macrophage marker interleukin-6 (IL-6), but not tumour necrosis factor (TNF)- $\alpha$ or monocyte chemotactic protein-1 (MCP-1), was significantly up-regulated (Table 3).

The protein expression of cell adhesion molecules ICAM-1 (fig. 1, J and K) and VCAM-1 (fig. 1M,N) was significantly increased in liver sections of Akita versus wild-type mice

Table 2.

Effect of rosiglitazone (RGZ) treatment on liver enzymes of Akita mice.

\begin{tabular}{|c|c|c|c|c|c|c|}
\hline & \multicolumn{2}{|c|}{ WT } & \multicolumn{2}{|c|}{ Akita } & \multicolumn{2}{|c|}{ Akita + RGZ } \\
\hline & Start & 4 months & Start & 4 months & Start & 4 months \\
\hline Alkaline phosphatases (U/l) & $227 \pm 10$ & $178 \pm 29$ & $662 \pm 14^{2}$ & $343 \pm 21^{2^{3}}$ & $543 \pm 37^{1^{2}}$ & $683 \pm 73^{1^{2}}$ \\
\hline $\mathrm{CRP}(\mathrm{ng} / \mathrm{ml})$ & $56 \pm 6.9$ & $129 \pm 16^{3}$ & $83 \pm 6.9^{2}$ & $165 \pm 23^{3}$ & $99 \pm 11^{2}$ & $152 \pm 20$ \\
\hline AST (U/l) & $85 \pm 8.1$ & $167 \pm 22^{3}$ & $111 \pm 8.4$ & $382 \pm 57^{2^{3}}$ & $128 \pm 9.7^{2}$ & $201 \pm 28^{1^{3}}$ \\
\hline $\mathrm{ALT}(\mathrm{U} / \mathrm{l})$ & $37 \pm 5.4$ & $102 \pm 24^{3}$ & $36 \pm 3.3$ & $356 \pm 69^{2^{3}}$ & $50 \pm 10$ & $155 \pm 30^{1^{3}}$ \\
\hline
\end{tabular}

Data are means \pm S.E.M. of 6 determinations.

${ }^{1} p \leq 0.05$ versus untreated Akita mice.

${ }^{2} p<0.05$ versus wild-type (WT) mice.

${ }^{3} p<0.05$ versus start.

Aspartate aminotransferases (AST), alanine aminotransferases (ALT) and C-reactive protein (CRP). 

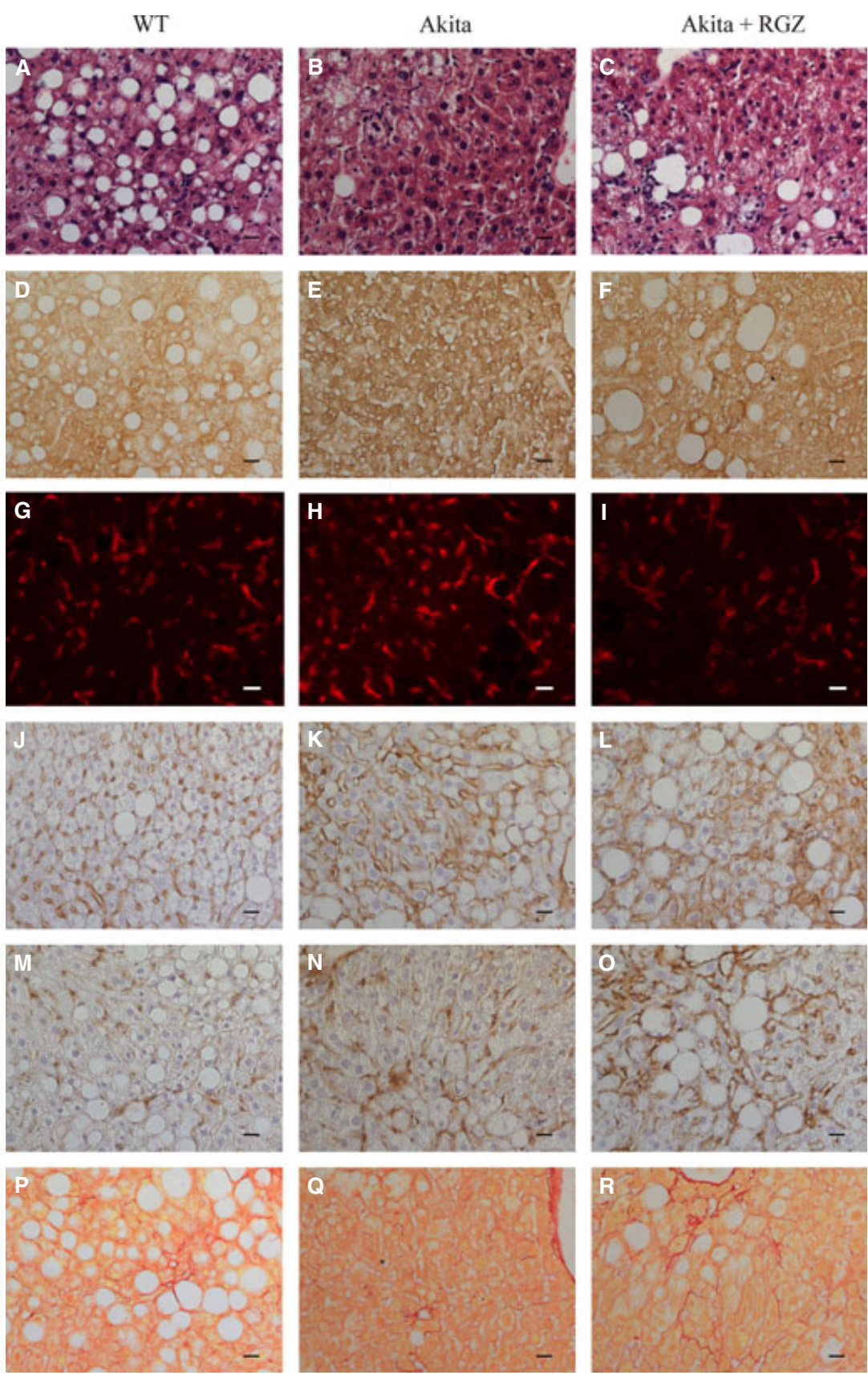

Fig. 1. Rosiglitazone (RGZ) induces steatosis but does not increase oxidative stress, inflammation or collagen deposition in diabetic Akita livers. Liver tissue of wild-type (WT) and Akita mice kept on a high-fat and high-cholesterol diet for 4 months, without or with addition of rosiglitazone at a daily dose of $10 \mathrm{mg} / \mathrm{kg}$ was immunohistologically analysed using different stainings: (A, B, C) Haematoxylin/eosin staining; (D, E, F) 3-nitrotyrosine staining; (G, H, I) F4/80 staining; (J, K, L) intercellular adhesion molecule-1 staining; (M, N, O) vascular cell adhesion molecule-1 staining; (P, Q, R) Sirius Red staining. The scale bars correspond to $20 \mu \mathrm{m}$. Magnification: $400 \times$.

(Table 4). Gene expression of ICAM-1 and VCAM-1, however, was not affected by genotype. With respect to genes involved in $\beta$-oxidation of fatty acids, the mRNA expression levels of fatty acid transport protein and medium-chain acylCoA dehydrogenase were not affected by genotype (Table 3 ).

Staining of liver sections with Sirius Red (fig. 1P,Q) did not indicate marked effects of genotype on collagen deposition that was located mainly around the blood vessels. Quantitative analysis confirmed this observation: stained area of
$2.1 \pm 0.39 \%$ for Akita mice versus $2.1 \pm 0.096 \%$ for wildtype mice on the same diet (Table 4).

Wild-type and Akita livers were not cholestatic as no hyperplasia of the CK-19 positive biliary ductules was found in the periportal region of the acinus (data not shown).

Effect of treatment. Treatment of Akita mice, kept on high-fat and high-cholesterol diet for 4 months, with rosiglitazone 
Table 3.

Effect of rosiglitazone (RGZ) treatment on gene expression in the liver of Akita mice.

\begin{tabular}{|c|c|c|c|}
\hline & WT & Akita & Akita + RGZ \\
\hline \multicolumn{4}{|l|}{ Steatosis } \\
\hline SREBP1c & $6.2 \pm 0.20$ & $5.5 \pm 0.23$ & $6.1 \pm 0.21$ \\
\hline PPAR- $\alpha$ & $0.87 \pm 0.15$ & $1.7 \pm 0.20^{2}$ & $1.8 \pm 0.23^{2}$ \\
\hline PPAR- $\gamma 1$ & $6.7 \pm 0.16$ & $6.8 \pm 0.15$ & $7.1 \pm 0.12^{2}$ \\
\hline PPAR- $\gamma 2$ & $8.5 \pm 0.36$ & $11 \pm 0.44^{2}$ & $8.9 \pm 0.11^{1}$ \\
\hline CD36 & $1.9 \pm 0.22$ & $1.2 \pm 0.19$ & $1.3 \pm 0.074^{2}$ \\
\hline FAS & $6.3 \pm 0.14$ & $6.9 \pm 0.32$ & $7.3 \pm 0.14^{2}$ \\
\hline \multicolumn{4}{|c|}{ Oxidative stress } \\
\hline Cat & $-2.4 \pm 0.096$ & $-1.9 \pm 0.33$ & $-1.7 \pm 0.11^{2}$ \\
\hline SOD1 & $-0.10 \pm 0.10$ & $-0.005 \pm 0.19$ & $0.56 \pm 0.13^{1^{2}}$ \\
\hline Gpx1 & $-1.7 \pm 0.12$ & $-1.5 \pm 0.20$ & $-1.1 \pm 0.15^{2}$ \\
\hline Xdh1 & $2.6 \pm 0.11$ & $2.3 \pm 0.17$ & $3.0 \pm 0.23^{1}$ \\
\hline Nox4 & $5.7 \pm 0.14$ & $6.4 \pm 0.32$ & $6.5 \pm 0.21^{2}$ \\
\hline iNOS & $14 \pm 0.33$ & $13 \pm 0.39^{2}$ & $12 \pm 0.26^{2}$ \\
\hline eNOS & $9.2 \pm 0.15$ & $8.7 \pm 0.18^{2}$ & $9.1 \pm 0.13$ \\
\hline \multicolumn{4}{|c|}{ Macrophages } \\
\hline Arg1 & $-0.69 \pm 0.13$ & $-1.7 \pm 0.23^{2}$ & $0.10 \pm 0.20^{1^{2}}$ \\
\hline Mrc1 & $5.6 \pm 0.14$ & $5.2 \pm 0.27$ & $5.4 \pm 0.16$ \\
\hline TNF- $\alpha$ & $12 \pm 0.33$ & $12 \pm 0.53$ & $11 \pm 0.18^{2}$ \\
\hline MCP-1 & $9.9 \pm 0.39$ & $9.3 \pm 0.51$ & $9.3 \pm 0.40$ \\
\hline IL-6 & $15 \pm 0.12$ & $14 \pm 0.30^{2}$ & $16 \pm 0.48^{1}$ \\
\hline F4/80 & $8.4 \pm 0.11$ & $6.9 \pm 0.29^{2}$ & $6.8 \pm 0.14^{2}$ \\
\hline Csf1r & $4.7 \pm 0.18$ & $3.9 \pm 0.31^{2}$ & $4.0 \pm 0.12^{2}$ \\
\hline CD68 & $13 \pm 0.17$ & $13 \pm 0.14^{2}$ & $13 \pm 0.15$ \\
\hline \multicolumn{4}{|c|}{ Cell adhesion } \\
\hline ICAM-1 & $6.6 \pm 0.25$ & $5.6 \pm 0.19$ & $6.9 \pm 0.15^{1}$ \\
\hline VCAM-1 & $6.6 \pm 0.085$ & $5.5 \pm 0.39$ & $5.9 \pm 0.11^{2}$ \\
\hline \multicolumn{4}{|c|}{$\beta$-oxidation of fatty acids } \\
\hline FATP & $5.8 \pm 0.23$ & $5.8 \pm 0.26$ & $6.8 \pm 0.079^{1^{2}}$ \\
\hline MCAD & $-0.53 \pm 0.16$ & $-0.16 \pm 0.40$ & $0.22 \pm 0.078^{2}$ \\
\hline
\end{tabular}

Data are means \pm S.E.M. of 6 measurements and represent gene expression values normalized to the housekeeping gene $\beta$-actin $(\Delta \mathrm{Ct}$ values).

${ }^{1} p<0.05$ versus untreated Akita mice.

${ }^{2} p<0.05$ versus wild-type (WT).

Abbreviations are defined in Table 1 .

Table 4.

Quantitation of immunohistochemical analysis of liver sections.

\begin{tabular}{lccc}
\hline & \multicolumn{3}{c}{ \% stained area } \\
\cline { 2 - 4 } & \multicolumn{1}{c}{ WT } & Akita & Akita + RGZ \\
\hline 3-NT & $42 \pm 3.5$ & $53 \pm 1.3$ & $60 \pm 3.3^{1}$ \\
F4/80 & $6.0 \pm 0.73$ & $9.5 \pm 2.2$ & $14 \pm 4.1$ \\
Mac-3 & $1.2 \pm 0.081$ & $2.0 \pm 0.29^{1}$ & $2.5 \pm 0.27^{1}$ \\
ICAM-1 & $22 \pm 2.4$ & $33 \pm 2.0^{1}$ & $28 \pm 1.4$ \\
VCAM-1 & $4.3 \pm 0.86$ & $7.8 \pm 1.2^{1}$ & $6.6 \pm 0.63$ \\
Sirius Red & $2.1 \pm 0.096$ & $2.1 \pm 0.39$ & $1.6 \pm 0.19$ \\
\hline
\end{tabular}

Data are means \pm S.E.M. of 6 determinations.

${ }^{1} p<0.05$ versus wild-type (WT).

Abbreviations are 3-nitrotyrosine (3-NT), intercellular adhesion molecule 1 (ICAM-1) and vascular cell adhesion molecule 1 (VCAM-1).

$(10 \mathrm{mg} / \mathrm{kg}$ per day) resulted in enhanced total body-weight (24 $\pm 0.56 \mathrm{~g} ; p<0.05)$ as compared to untreated Akita mice. Liver weight was also enhanced upon rosiglitazone treatment $(2,326 \pm 99 \mathrm{mg}$ versus $1,492 \pm 71 \mathrm{mg}$ in untreated Akita mice), corresponding to $9.9 \pm 0.54 \%$ and $8.0 \pm 0.21 \%$ of total body-weight, respectively $(p<0.01)$. Food intake was comparable in both groups (not shown). Rosiglitazone treatment resulted in markedly elevated levels of alkaline phosphatases and reduced levels of AST and ALT as compared to untreated Akita mice on the same diet (Table 2). Rosiglitazone treatment of diabetic Akita mice was associated with enhanced lipid accumulation in the liver, as shown by H\&E staining (fig. 1B,C). Furthermore, triglyceride levels were significantly elevated in liver extracts of rosiglitazonetreated mice $\left(15 \pm 1.3 \mathrm{mg} \times \mathrm{dL}^{-1} / \mathrm{mg}\right.$ tissue versus $8.2 \pm$ $1.7 \mathrm{mg} \times \mathrm{dL}^{-1} / \mathrm{mg}$ tissue; $\left.p=0.03\right)$ and were comparable with wild-type mice $\left(18 \pm 1.9 \mathrm{mg} \times \mathrm{dL}^{-1} / \mathrm{mg}\right.$ tissue). Liver gene expression of the steatosis markers SREBP1c, PPAR- $\alpha$, CD36 and fatty acid synthase was, however, not significantly affected by rosiglitazone treatment (Table 3).

Expression analysis in liver tissue of anti-oxidant genes revealed significant down-regulation by rosiglitazone treatment of SOD1, whereas the expression of Cat and Gpx1 was not markedly affected (Table 3). Of the pro-oxidant genes analysed, only Xdh1 was down-regulated, whereas Nox-4, iNOS and eNOS were not significantly affected. Staining of liver tissue sections for the oxidative stress marker 3-NT did not indicate marked effects of rosiglitazone treatment (fig. 1, E and F). Quantitative analysis confirmed this finding (Table 4).

Monitoring of macrophage content in liver tissues by staining for F4/80 (fig. 1, H and I) or Mac-3 (not shown) did not reveal marked effects of rosiglitazone treatment. Stained areas for rosiglitazone-treated and untreated Akita livers were comparable but somewhat enhanced as compared to wild-type samples (Table 4). This was compatible with unaltered gene expression of the general macrophage markers F4/80, Csf1r and CD68 (Table 3). Relative gene expression of the M2 macrophage marker Arg1, but not of Mrc1, was significantly down-regulated upon rosiglitazone treatment. In addition, the M1 macrophage marker IL-6, but not TNF- $\alpha$ or MCP-1, was significantly down-regulated (Table 3 ).

Staining of liver sections for the cell adhesion molecules ICAM-1 (fig. 1K,L) and VCAM-1 (fig. 1N,O) did not reveal significant effects of rosiglitazone. Stained areas of rosiglitazone-treated versus untreated livers were comparable (Table 4). Gene expression of ICAM-1 was markedly up-regulated by rosiglitazone treatment of Akita mice, whereas that of VCAM-1 was not affected. With respect to genes involved in $\beta$-oxidation of fatty acids, we observed significantly reduced expression of fatty acid transport protein in the liver of rosiglitazone-treated mice as compared to untreated Akita or wild-type mice (Table 3). Expression of medium-chain acyl-CoA dehydrogenase in Akita mice was not affected by treatment. The plasma level of the acute phase reactant C-reactive protein increased with the high-fat and high-cholesterol diet in both groups, but was not affected by rosiglitazone treatment (Table 2).

Staining of liver sections with Sirius Red (fig. 1Q,R) did not show an effect of rosiglitazone treatment on hepatic collagen content. Quantitative analysis confirmed this observation: stained area of $1.6 \pm 0.19 \%$ versus $2.1 \pm 0.39 \%$ for untreated Akita mice on the same diet (Table 4). 
Rosiglitazone treatment did not induce cholestatic liver injury as no biliary duct hyperplasia was detected in rosiglitazone-treated Akita livers (data not shown).

\section{Discussion}

Troglitazone, the first-generation drug of the thiazolidinedione class to treat type 2 diabetes, was withdrawn from the market due to several reports of hepatotoxicity. For the second-generation thiazolidinediones, rosiglitazone and pioglitazone, hepatotoxicity was only reported in some patients with enlarged livers, associated with an increased fat accumulation (hepatosteatosis) and occasionally with liver failure [10,11,20-25]. The mechanism of rosiglitazone-induced liver injury is still unclear and unpredictable. In general, rosiglitazone has been well tolerated in patients with inadequately controlled type 2 diabetes suffering from non-alcoholic fatty liver disease. In addition, liver function improved in about $30 \%$ of these patients after treatment [26].

Diabetic Akita mice, kept on a high-fat and high-cholesterol diet for 4 months, already showed rosiglitazone-induced side effects such as an increased weight and fat mass gain upon treatment with $10 \mathrm{mg} / \mathrm{kg}$ per day rosiglitazone [16]. In addition, treatment of these mice with a higher dose of $30 \mathrm{mg} / \mathrm{kg}$ per day rosiglitazone induced left ventricular hypertrophy and early signs of heart failure [16]. Administration of rosiglitazone at a dose of $10 \mathrm{mg} / \mathrm{kg}$ per day to Akita mice was previously shown to be associated with enhanced plasma adiponectin levels, decreased glucose levels and increased insulin sensitivity, confirming the in vivo efficacy of rosiglitazone in this model [16]. In this study, the effect of rosiglitazone treatment on liver structure and function of diabetic Akita mice was investigated by determining the stage of non-alcoholic fatty liver disease ranging from hepatosteatosis (which is relatively benign) to hepatic inflammation, fibrosis and ultimately progressing to cirrhosis. Rosiglitazone treatment of Akita mice did not affect liver expression of PPAR- $\gamma_{1}$, whereas that of PPAR- $\gamma_{2}$ was up-regulated to the level of wild-type mice.

Rosiglitazone treatment induced hepatosteatosis. Histological analysis of liver sections showed more steatosis in treated as compared to control Akita mice. This was confirmed by an elevated triglyceride concentration in liver extracts. GarciaRuiz et al. [27] previously reported significantly enhanced steatosis and triglyceride content in the liver of $o b / o b$ mice treated with rosiglitazone. In addition, A-ZIP/F-1 'fatless' mice, in essence lacking white adipose tissue, have a $30 \%$ increased liver triglyceride and fatty acyl-CoA content upon a 3-week rosiglitazone (3 $\mathrm{mg} / \mathrm{kg}$ per day) administration [28]. A fatty liver (non-alcoholic hepatosteatosis) is characterized by elevated circulating levels of ALT and AST, markers of hepatocellular damage. In this study, however, plasma levels of the liver enzymes ALT and AST showed a lower trend in the treated versus the untreated group, suggesting a protective effect of rosiglitazone against high-fat and high-cholesterol diet-induced liver damage (as assessed by increased liver ALT and AST levels after 4 months diet, Table 2). ALP levels were, however, significantly increased, suggesting induction of liver injury. Frequently, the ALP test is used to detect blockage of bile ducts. However, bile duct hyperplasia after cholestasis liver injury was not detected in liver sections of rosiglitazone-treated versus untreated Akita animals (data not shown).

The molecular mechanism of hepatic steatosis in the Akita mice treated with rosiglitazone may be explained by the reduced gene expression of fatty acid transport protein. However, untreated Akita and wild-type mice have a similar liver fatty acid transport protein gene expression, although there is clearly less hepatic lipid accumulation in the untreated Akita versus wild-type mice. A possible explanation for this observation may be due to the increased energy expenditure seen in Akita versus wild-type mice [14]. This increased energy consumption has been suggested to result from an enhanced whole-body lipid oxidation secondary to hypoinsulinaemia as seen in patients with type 1 diabetes [29]. The relative gene expression levels of other steatosis markers, such as PPAR- $\alpha$, a transcription factor for free fatty acid oxidation [7], and CD36, a scavenger receptor involved in free fatty acid uptake, were, however, comparable for the treated and the untreated mice. In addition, the relative mRNA expression levels of SREBP1c, a transcription factor involved in de novo lipogenesis, and fatty acid synthase, a catalyst for fatty acid synthesis, were lower in the treated group as compared to the controls, suggesting that these observed changes in gene expression do not contribute to the enhanced liver steatosis in rosiglitazonetreated mice. Gupte et al. [30] in contrast found increased SREBP1c and fatty acid synthase expression upon rosiglitazone treatment of low-density lipoprotein receptor deficient mice. In a previous study [31], Otsuka-Long Evans-Tokushima fatty rats, which develop diabetes, obesity and non-alcoholic fatty liver disease, were treated with rosiglitazone $(4 \mathrm{mg}$ / $\mathrm{kg}$ during 28 weeks). Treatment resulted in mild improvement of liver steatosis and was associated with enhanced expression of the fatty acid $\beta$-oxidation enzymes fatty acid transport protein and medium-chain acyl-CoA dehydrogenase.

As oxidative stress or an imbalance between pro- and antioxidant pathways can promote the transition from benign steatosis to steatohepatitis as part of the 'second hit' hypothesis of non-alcoholic steatohepatitis, the gene expression of several pro- and anti-oxidant markers was examined in the liver of rosiglitazone-treated and untreated Akita mice. Apparently conflicting data were obtained. Indeed, whereas the anti-oxidant SOD1 showed a decreased gene expression, suggesting an increased oxidative stress, the pro-oxidant Xdh1 was also down-regulated suggesting reduced oxidative stress. Expression of eNOS, which can be down-regulated by reactive oxygen species (ROS) [32], was not significantly affected by rosiglitazone treatment, and 3-NT determination further did not reveal differences in oxidative stress. Although no difference was found in 3-NT levels, Garcia-Ruiz et al. [27], in contradiction to our findings, found an increase in oxidative stress (measured from lipid peroxidation) in the liver of $o b / o b$ 
mice treated with $1 \mathrm{mg} / \mathrm{kg}$ body-weight per day rosiglitazone. This increase in liver oxidative stress in rosiglitazone-treated $o b / o b$ mice may be related to an enhanced mitochondrial function, which results in the generation of more ROS. Therefore, it cannot be excluded that the balance between pro- and antioxidant actions favours oxidative stress in the liver of rosiglitazone-treated Akita mice, although not detected by 3-NT staining.

Rosiglitazone treatment did not cause steatohepatitis. In obese patients with type 2 diabetes, an elevated secretion of inflammatory cytokines by adipocytes leads to activation of Kupffer cells (M1 phenotype), hepatocytes and stellate cells. In hepatocytes, IL-6 can induce the expression of C-reactive protein. C-reactive protein is an acute phase protein, which stimulates endothelial cells to produce inflammatory adhesion molecules, such as ICAM-1 and VCAM-1. In turn, these components are involved in the transmigration of monocytes and macrophages to the site of injury [33,34]. In our study, the plasma level of C-reactive protein, however, was not different between both groups, in contrast to other animal and human studies, which reported a reduction of plasma C-reactive protein $[33,35]$. As macrophages and cell adhesion molecules play an important role in inflammation, these were further investigated. Rosiglitazone treatment did not alter gene expression of F4/80, CD68 or csf1r, and the total liver macrophage content (F4/80- and Mac-3-positive cells) in treated and untreated mice was similar. An increase in ROS can switch the polarization of Kupffer cells from an anti-inflammatory M2 to a pro-inflammatory M1 phenotype; whereas M2 macrophages act as scavenger molecules and repair damaged tissues, M1 macrophages produce proinflammatory cytokines and are involved in tissue destruction [36]. However, in the Akita mice, both the M2 macrophage marker Arg1 and the M1 macrophage marker IL-6 showed a decreased gene expression in the treated animals, indicating no enrichment in a specific macrophage subpopulation. The expression levels of the cell adhesion molecules, however, suggest a slight decrease in inflammation after rosiglitazone treatment: ICAM-1 gene expression was significantly lower and VCAM-1 gene and ICAM-1 protein expression showed a lower trend in the treated versus untreated group. As VCAM-1 and ICAM-1 are required for the recruitment of inflammatory cells to sites of injury, reduced expression may be associated with a lower inflammatory response.

Rosiglitazone treatment did not cause steatofibrosis. Patients with type 2 diabetes have a higher risk of developing steatofibrosis [37]. Liver fibrosis is caused by the accumulation of extracellular matrix materials, such as collagen, generated by activated hepatic stellate cells and myofibroblasts. Activated Kupfer cells, infiltrating monocytes, activated aggregated platelets and damaged hepatocytes trigger intracellular signalling cascades that lead to activation of the stellate cells $[38,39]$. Histological analysis of liver sections, however, showed no additional collagen deposition in the rosiglitazone-treated group. Collagen deposition was mostly found around the blood vessels, while in non-alcoholic fatty liver disease patients, fibrosis is found around the blood vessels and perisinusoidally. Thus, Akita mice did not show signs of steatofibrosis and rosiglitazone treatment did not induce this.

In conclusion, in this study, we investigated the effects of the thiazolidinedione rosiglitazone $(10 \mathrm{mg} / \mathrm{kg}$ body-weight per day) on the different stages of non-alcoholic fatty liver disease in genetically diabetic Akita mice, kept on a high-fat and high-cholesterol diet for 4 months. An increase in steatosis in the liver was observed, which may be partially responsible for the increased liver weight in mice treated with rosiglitazone. This effect is similar to the steatosis seen in other mouse models (ob/ob mice and A-ZIP/F-1 fatless mice) and may explain a potential mechanism in rare cases of rosiglitazone-induced hepatosteatosis in patients. The progression of steatosis to the more severe stages of non-alcoholic fatty liver disease, steatohepatitis and steatofibrosis (which occurs rarely in rosiglitazone-treated patients) was, however, not observed at the dose of rosiglitazone tested in the present study. As rosiglitazone also significantly lowered the plasma level of markers for hepatocellular damage (AST and ALT) and tended to decrease liver inflammation, the rosiglitazone-induced liver steatosis in Akita mice may provide a protection of peripheral tissues such as muscle and pancreas against the deleterious effects of dietary free fatty acid.

\section{Acknowledgements}

Skilful technical assistance by L. Frederix, A. De Wolf, I. Vorsters and C. Vranckx is gratefully acknowledged. This study was supported financially by grants from Janssen Pharmaceutica NV and the FWO (G.0678.10N). The Center for Molecular and Vascular Biology is supported by the Programmafinanciering KU Leuven (PF/10/014).

\section{Conflict of interest}

The authors state that they have no conflicts of interest to disclose.

\section{References}

1 Angulo P. Nonalcoholic fatty liver disease. $N$ Engl J Med 2002;346:1221-31.

2 Clark JM, Brancati FL, Diehl AM. The prevalence and etiology of elevated aminotransferase levels in the United States. Am J Gastroenterol 2003;98:960-7.

3 Farrell GC, Larter CZ. Nonalcoholic fatty liver disease: from steatosis to cirrhosis. Hepatology 2006;43:S99-112.

4 Fujii H, Kawada N. Inflammation and fibrogenesis in steatohepatitis. J Gastroenterol 2012;47:215-25.

5 Ruhl CE, Everhart JE. Determinants of the association of overweight with elevated serum alanine aminotransferase activity in the United States. Gastroenterology 2003;124:71-9.

6 Nesto RW, Bell D, Bonow RO, Fonseca V, Grundy SM, Horton ES et al. Thiazolidinedione use, fluid retention, and congestive heart failure: a consensus statement from the American Heart Association and American Diabetes Association. October 7, 2003. Circulation 2003;108:2941-8. 
7 Abbas A, Blandon J, Rude J, Elfar A, Mukherjee D. PPARgamma agonist in treatment of diabetes: cardiovascular safety considerations. Cardiovasc Hematol Agents Med Chem 2012;10:12434.

8 Wang X, Liu X, Zhan Y, Lavallie ER, Diblasio-Smith L, CollinsRacie L et al. Pharmacogenomic, physiological, and biochemical investigations on safety and efficacy biomarkers associated with the peroxisome proliferator-activated receptor-gamma activator rosiglitazone in rodents: a translational medicine investigation. J Pharmacol Exp Ther 2010;334:820-9.

9 Laverty H, Benson C, Cartwright E, Cross M, Garland C, Hammond $\mathrm{T}$ et al. How can we improve our understanding of cardiovascular safety liabilities to develop safer medicines? $\mathrm{Br}$ J Pharmacol 2011;163:675-93.

10 Marcy TR, Britton ML, Blevins SM. Second-generation thiazolidinediones and hepatotoxicity. Ann Pharmacother 2004;38:141923.

11 Scheen AJ. Thiazolidinediones and liver toxicity. Diabetes Metab 2001;27:305-13.

12 Donato MT, Gomez-Lechon MJ. Drug-induced liver steatosis and phospholipidosis: cell-based assays for early screening of drug candidates. Curr Drug Metab 2012;13:1160-73.

13 Kleiner DE, Gaffey MJ, Sallie R, Tsokos M, Nichols L, McKenzie $\mathrm{R}$ et al. Histopathologic changes associated with fialuridine hepatotoxicity. Mod Pathol 1997;10:192-9.

14 Hong EG, Jung DY, Ko HJ, Zhang Z, Ma Z, Jun JY et al. Nonobese, insulin-deficient Ins2Akita mice develop type 2 diabetes phenotypes including insulin resistance and cardiac remodeling. Am J Physiol Endocrinol Metab 2007;293:E1687-96.

15 Yoshioka M, Kayo T, Ikeda T, Koizumi A. A novel locus, Mody4, distal to D7Mit189 on chromosome 7 determines early-onset NIDDM in nonobese C57BL/6 (Akita) mutant mice. Diabetes 1997;46:887-94.

16 Hemmeryckx B, Hoylaerts MF, Gallacher DJ, Rong Lu H, Himmelreich U, D'Hooge $\mathrm{J}$ et al. Does rosiglitazone affect adiposity and cardiac function in genetic diabetic mice? Eur $\mathrm{J}$ Pharmacol 2013;700:23-31.

17 Saxena NK, Ikeda K, Rockey DC, Friedman SL, Anania FA. Leptin in hepatic fibrosis: evidence for increased collagen production in stellate cells and lean littermates of ob/ob mice. Hepatology 2002;35:762-71.

18 Luttun A, Lutgens E, Manderveld A, Maris K, Collen D, Carmeliet $\mathrm{P}$ et al. Loss of matrix metalloproteinase- 9 or matrix metalloproteinase-12 protects apolipoprotein E-deficient mice against atherosclerotic media destruction but differentially affects plaque growth. Circulation 2004;109:1408-14.

19 Wong T, Hildebrandt MA, Thrasher SM, Appleton JA, Ahima RS, Wu GD. Divergent metabolic adaptations to intestinal parasitic nematode infection in mice susceptible or resistant to obesity. Gastroenterology 2007;133:1979-88.

20 Al-Salman J, Arjomand H, Kemp DG, Mittal M. Hepatocellular injury in a patient receiving rosiglitazone. A case report. Ann Intern Med 2000;132:121-4.

21 Bonkovsky HL, Azar R, Bird S, Szabo G, Banner B. Severe cholestatic hepatitis caused by thiazolidinediones: risks associated with substituting rosiglitazone for troglitazone. Dig Dis Sci 2002;47:1632-7.
22 Dhawan M, Agrawal R, Ravi J, Gulati S, Silverman J, Nathan G et al. Rosiglitazone-induced granulomatous hepatitis. J Clin Gastroenterol 2002;34:582-4.

23 Forman LM, Simmons DA, Diamond RH. Hepatic failure in a patient taking rosiglitazone. Ann Intern Med 2000;132:118-21.

24 Gouda HE, Khan A, Schwartz J, Cohen RI. Liver failure in a patient treated with long-term rosiglitazone therapy. Am J Med 2001;111:584-5.

25 Ravinuthala RS, Nori U. Rosiglitazone toxicity. Ann Intern Med 2000;133:658.

26 Wang $\mathrm{CH}$, Leung $\mathrm{CH}$, Liu SC, Chung CH. Safety and effectiveness of rosiglitazone in type 2 diabetes patients with nonalcoholic Fatty liver disease. J Formos Med Assoc 2006;105:743-52.

27 Garcia-Ruiz I, Rodriguez-Juan C, Diaz-Sanjuan T, Martinez MA, Munoz-Yague T, Solis-Herruzo JA. Effects of rosiglitazone on the liver histology and mitochondrial function in ob/ob mice. Hepatology 2007;46:414-23.

28 Kim JK, Fillmore JJ, Gavrilova O, Chao L, Higashimori T, Choi $\mathrm{H}$ et al. Differential effects of rosiglitazone on skeletal muscle and liver insulin resistance in A-ZIP/F-1 fatless mice. Diabetes 2003;52:1311-8.

29 Perseghin G, Lattuada G, De Cobelli F, Esposito A, Costantino F, Canu $\mathrm{T}$ et al. Reduced intrahepatic fat content is associated with increased whole-body lipid oxidation in patients with type 1 diabetes. Diabetologia 2005;48:2615-21.

30 Gupte AA, Liu JZ, Ren Y, Minze LJ, Wiles JR, Collins AR et al. Rosiglitazone attenuates age- and diet-associated nonalcoholic steatohepatitis in male low-density lipoprotein receptor knockout mice. Hepatology 2010;52:2001-11.

31 Seo YS, Kim JH, Jo NY, Choi KM, Baik SH, Park JJ et al. PPAR agonists treatment is effective in a nonalcoholic fatty liver disease animal model by modulating fatty-acid metabolic enzymes. J Gastroenterol Hepatol 2008;23:102-9.

32 Sharma A, Bernatchez PN, de Haan JB. Targeting endothelial dysfunction in vascular complications associated with diabetes. Int $\mathbf{J}$ Vasc Med 2012;2012:750126.

33 Pasceri V, Willerson JT, Yeh ET. Direct proinflammatory effect of C-reactive protein on human endothelial cells. Circulation 2000;102:2165-8.

34 Rattazzi M, Puato M, Faggin E, Bertipaglia B, Zambon A, Pauletto P. C-reactive protein and interleukin-6 in vascular disease: culprits or passive bystanders? J Hypertens 2003;21:1787-803.

35 Qayyum R, Adomaityte J. Meta-analysis of the effect of thiazolidinediones on serum C-reactive protein levels. Am J Cardiol 2006;97:655-8.

36 Day CP. Pathogenesis of steatohepatitis. Best Pract Res Clin Gastroenterol 2002;16:663-78.

37 Smith BW, Adams LA. Nonalcoholic fatty liver disease and diabetes mellitus: pathogenesis and treatment. Nat Rev Endocrinol 2011;7:456-65.

38 Kershenobich Stalnikowitz D, Weissbrod AB. Liver fibrosis and inflammation. A review. Ann Hepatol 2003;2:159-63.

39 Khandoudi N, Delerive P, Berrebi-Bertrand I, Buckingham RE Staels B, Bril A. Rosiglitazone, a peroxisome proliferator-activated receptor-gamma, inhibits the Jun $\mathrm{NH}(2)$-terminal kinase/activating protein 1 pathway and protects the heart from ischemia/reperfusion injury. Diabetes 2002;51:1507-14. 